\title{
1 Map7D2 and Map7D1 facilitate MT stabilization through distinct 2 mechanisms to control cell motility and neurite outgrowth
}

3 Koji Kikuchi ${ }^{*}$, Yasuhisa Sakamoto ${ }^{1}$, Akiyoshi Uezu², Hideyuki Yamamoto ${ }^{3}$, Kei-ichiro

4 Ishiguro $^{4}$, Kenji Shimamura ${ }^{5}$, Taro Saito ${ }^{6}$, Shin-ichi Hisanaga ${ }^{6}$, and Hiroyuki Nakanishi ${ }^{1^{*}}$

$5 \quad{ }^{1}$ Department of Molecular Pharmacology, Graduate School of Medical Sciences, Kumamoto

6 University, 1-1-1 Honjo, Kumamoto 860-8556, Japan

$7 \quad{ }^{2}$ Department of Cell Biology, Duke University Medical School, Durham, North Carolina 27710,

$8 \quad$ U.S.A

$9{ }^{3}$ Department of Biochemistry, Graduate School of Medicine, University of the Ryukyus, 10 Okinawa 903-0215, Japan.

$11{ }^{4}$ Department of Germline Development, Institute of Molecular Embryology and Genetics, 12 Kumamoto University, 2-2-1 Honjo, Chuo-ku, Kumamoto 860-0811, Japan

$13{ }^{5}$ Department of Brain Morphogenesis, Institute of Molecular Embryology and Genetics, 14 Kumamoto University, 2-2-1 Honjo, Chuo-ku, Kumamoto 860-0811, Japan

$15{ }^{6}$ Department of Biological Sciences, Tokyo Metropolitan University, Hachioji, Tokyo 192-0397, 16 Japan

*Correspondence: kojik@kumamoto-u.ac.jp (K.K), hnakanis@gpo.kumamoto-u.ac.jp (H. N.)

20 Keywords: Map7D2/Map7D1/Microtubule stabilization/Cell motility/Neurite outgrowth 


\section{Abstract}

2 Microtubule (MT) dynamics are modulated through the coordinated action of various

3 MT-associated proteins (MAPs). However, the regulatory mechanisms underlying MT

4 dynamics remain unclear. Herein, we show that MAP7 family protein Map7D2 facilitates MT

5 stabilization to control cell motility and neurite outgrowth. Map7D2, was highly expressed in

6 the brain and testis, directly bound to MTs through its N-terminal half similarly to Map7, and

7 promoted MT stabilization in vitro. Map7D2 localized prominently to the centrosome and

8 partially on MTs in N1-E115 mouse neuroblastoma cells, which expresses two of the four

9 MAP7 family members, Map7D2 and Map7D1. Map7D2 loss decreased the intensity of MTs

10 without affecting stable MT markers acetylated and detyrosinated tubulin, suggesting that

11 Map7D2 stabilizes MTs via direct binding. In addition, Map7D2 loss increased the rate of

12 random cell migration and neurite outgrowth, presumably by disturbing the balance between

13 MT stabilization and destabilization. The other MAP7 family protein expressed in N1-E115,

14 Map7D1, exhibited similar subcellular localization and gene knock-down phenotypes. However,

15 in contrast to Map7D2, Map7D1 was required for the maintenance of acetylated tubulin levels.

16 Taken together, our data suggest that Map7D2 and Map7D1 facilitate MT stabilization through

17 distinct mechanisms for the control of cell motility and neurite outgrowth. 


\section{Introduction}

2 Microtubule (MT) dynamics play crucial roles in a variety of cellular processes, including

3 mitosis, vesicle and organelle transport, as well as cell motility and morphology (Cleary, 2021;

4 Etienne-Manneville, 2013; Roll-Mecak, 2020). MT dynamics are altered in response to various

5 intrinsic or extrinsic signals and are then modulated through the coordinated actions of various

6 MT-associated proteins (MAPs), which control the processes of dynamic instability (Cleary,

7 2021; Roll-Mecak, 2020). Therefore, it is important to identify and characterize MAPs in order

8 to understand the regulatory mechanisms of MT dynamics. We previously performed a

9 comprehensive proteomic analysis of MT co-sedimented proteins from the brain and identified

10 a series of functionally uncharacterized MT-binding proteins (Sakamoto, 2008). The list

11 included MAP7 family members Map7, Map7D1, and Map7D2, but not Map7D3. Among the

12 MAP7 family, Map7 has been extensively characterized. Several lines of evidence suggest that

13 Map7 has the ability to stabilize and reorganize MTs. Ectopic expression of Map7 induces MT

14 bundling and resistance to nocodazole treatment-induced MT depolymerization (Masson, 1993).

15 Map7 expression is upregulated during MT reorganization in response to the differentiation of

16 keratinocytes (Fabre-Jonca, 1999) and the establishment of apicobasal polarity in human colon

17 adenocarcinoma cell lines such as Caco-2 and HT-29-D4 cells (Carles, 1999; Masson, 1993). In

18 addition, recent studies have shown that Map7 and the Drosophila Map7 homolog, Ensconsin

19 (Ens), are involved in Kinesin-1-dependent transport by promoting the recruitment of a

20 conventional Kinesin-1, Kif5b, and its Drosophila homolog, Khc, to MTs during various

21 biological processes (Barlan, 2013; Hooikaas, 2019; Kikuchi, 2018; Metzger, 2012; Sung, 2008;

22 Tymanskyj, 2018). The competition between Map7 and other MAPs for MT binding regulates

23 the loading of motor proteins, thereby controlling the distribution and balance of motor activity

24 in neurons (Monroy, 2018; Monroy, 2020). While a considerable body of evidence has

25 highlighted the important roles of Map7 in the regulation of MT dynamics, the function of

26 MAP7 family member Map7D2 in the regulation of MT dynamics and its relationship with

27 other MAP7 family members remain unclear.

28 In this study, we determined the tissue distribution and biochemical properties of 29 Map7D2 for the first time. Map7D2 is expressed predominantly in the glomerular layer of the 30 olfactory bulb and the Sertoli cells of testes. Further, it directly associates with MTs through its

31 N-terminal half, similarly to Map7, significantly enhancing MT stabilization. We also examined

32 the cellular functions of Map7D2 using the N1-E115 mouse neuroblastoma cell line, which 
1 expresses both Map7D2 and Map7D1, but not Map7 nor Map7D3. Map7D2 predominantly

2 localizes to the centrosome and partially on MTs, and suppresses cell motility and neurite

3 outgrowth by facilitating MT stabilization via direct binding. Finally, we determined the

4 functional differences between Map7D2 and Map7D1 with regard to MT stabilization in

$5 \quad$ N1E115 cells. Although Map7D1 exhibits similar subcellular localization and gene knock-down

6 phenotypes to Map7D2, Map7D1 is required to maintain the amount of acetylated tubulin in

7 contrast to Map7D2. These results suggest that Map7D2 and Map7D1 facilitate MT

8 stabilization through distinct mechanisms for the control of cell motility and neurite outgrowth.

\section{$9 \quad$ Results}

10 Map7D2 is highly expressed in the glomerular layer of the olfactory bulb and the Sertoli 11 cells of testes.

12 We previously performed a comprehensive proteomic analysis of MT co-sedimented proteins

13 from the rat brain and identified a number of novel factors (Sakamoto, 2008). In the present 14 study, we focused on Map7D2, which is one of the MAP7 family members (Fig. S1A). To 15 analyze the tissue distribution of Map7D2, we first performed northern blotting analysis using 16 total RNA extracted from various rat tissues. Northern blotting analysis showed that the 17 approximately 4.2-kb mRNA was hybridized only in the brain and testis, being more abundant 18 in the former (Fig. 1A). Of note, no detectable signal was observed in other rat tissues examined, 19 including the heart, spleen, lung, liver, skeletal muscle, and kidney. Next, we investigated the 20 tissue distribution of Map7D2 at the protein level by immunoblotting. For the immunoblotting 21 analysis, we raised an anti-Map7D2 polyclonal antibody using aa 1-235 of rat Map7D2 22 (rMap7D2) as an epitope (Fig. S2A). Using lysates from HeLa cells transfected with an empty 23 vector, hMap7-V5His 6 , or rMap7D2-V5His 6 , we confirmed that the antibody detected Map7D2, 24 but not Map7 (Fig. S2A). In addition, we evaluated antibody specificity by siRNA-mediated 25 knock-down of endogenous Map7D2. For this experiment, we used a mouse neuroblastoma cell 26 line, N1-E115, in which the expression of Map7d2 and Map7d1, but not Map7 and Map7d3, 27 was detected by quantitative real-time PCR (RT-qPCR) (Fig. S2B). We designed three 28 independent siRNAs against Map $7 d 2$ or Map7d1. The immunoreactive band disappeared 29 following treatment with each Map7d2 siRNA, but not the control or Map7d1 siRNA (Fig. 30 S2C), indicating that the antibody specifically recognized Map7D2. We then performed 31 immunoblotting analysis using lysates from various rat tissues. Consistent with the northern 
1 blotting analysis, Map7D2 was detected at the protein level only in the brain and testis, while no

2 immunoreactive bands were detected in other rat tissues (Fig. 1B).

3 We further analyzed the expression patterns of Map7D2 in the brain and testis by 4 immunofluorescence. Based on RNA-seq CAGE, RNA-Seq, and SILAC database analysis (Fig.

5 S3; Expression Atlas, https://www.ebi.ac.uk/gxa/home/), Map7D2 expression was confirmed in

6 the four brain tissue regions of postnatal day 0 mice, the cerebellum, cerebral cortex,

7 hippocampus, and olfactory bulb. Among these regions, Map7D2 was most highly expressed in 8 the Map2-negative area of the olfactory bulb, the glomerular layer (Fig. 1C). Only weak signals

9 were detected in the cerebellum, cerebral cortex, and hippocampus (Fig. 1C). Next, we analyzed

10 Map7D2 expression in the seminiferous tubules of adult mice. Map7D2 signals were merged

11 with Tubb3 signals, a marker for Sertoli cells (Fig. 1C), indicating that Map7D2 is expressed

12 predominantly in Sertoli cells. Taken together, these data suggest that in vivo, Map7D2 may

13 function in the glomerular layer of the olfactory bulb and the Sertoli cells of the testis.

Map7D2 has an ability to stabilize MTs.

MAP7 family members share two conserved regions (Fig. S1A). The amino acid sequences of the N-terminal (aa 53-138) and C-terminal (aa 389-562) regions of human Map7D2 (hMap7D2) were $64.0 \%$ and $42.9 \%$ identical to those of human Map7 (hMap7), respectively, while other regions showed no significant homology to hMap7 (Fig. 2A). Using the rat brain cDNA library, we obtained rMap7D2 cDNA by PCR. The cloned cDNA encoded a protein consisting of 763 aa with a molecular weight of 84,823 (DDBJ/EMBL/GenBank accession number AB266744) (Fig. 2A). The full-length aa sequence of rMap7D2 was $68.1 \%$ identical to that of hMap7D2. For subsequent experiments, we used the rMap7D2 that we cloned.

We sought to determine whether rMap7D2 directly binds to MTs. To this end, we performed an MT co-sedimentation assay using recombinant rMap7D2. When His 6 -rMap7D2 was incubated with MTs, followed by ultracentrifugation, it was recovered with MTs in the pellet (Fig. 2B). The dissociation constant (Kd) was calculated to be approximately $6 \times 10^{-7} \mathrm{M}$ (Fig. 2B). This value is comparable to those of well-known MAPs Tau and CLIP-170 (Gustke, 1994; Lansbergen, 2004). The stoichiometry of His 6 -rMap7D2 binding to tubulin was calculated 
1 comparable to that of Map7 (Bulinski, 1994). It has been reported that Map7 binds to MTs

2 through a conserved region on the $\mathrm{N}$-terminal side, while Map7D3 binds via a conserved region

3 on the C-terminal side (Yadav et al., 2014). To further examine the location of the MT-binding

4 domain of rMap7D2, the N-terminal (aa 1-421) and C-terminal (aa 422-763) halves were

$5 \quad$ subjected to an MT co-sedimentation assay (Fig. 2A). The N-terminal half was co-sedimented

6 with MTs, whereas the C-terminal half was not (Fig. 2C). These results indicate that the

$7 \quad$ MT-binding domain of rMap7D2 is located at the N-terminal half, similarly to that of Map7, but 8 not Map7D3.

9 Next, we tested whether rMap7D2 affects MT dynamics. The MT turbidity assay was 10 used to analyze the effect of rMap7D2 on the kinetics of MT assembly. The addition of 11 rMap7D2 significantly enhanced the amount of polymerized MTs in a time-dependent manner, 12 whereas tubulin self-polymerized even in the absence of rMap7D2 (Fig. 2D). Identical results 13 were observed by fluorescence microscopy analysis using rhodamine-labeled tubulin (Fig. 2E).

14 Furthermore, we investigated the ability of Map7D2 to bundle MTs in HeLa cells. Consistent 15 with the in vitro data, overexpression of Myc-rMap7D2 induced MT bundling in HeLa cells 16 (Fig. 2F). Taken together, these results indicate that Map7D2 facilitates MT stabilization.

\section{Map7D2 localized prominently to the centrosome and partially to MTs.}

19 Following the biochemical characterization of Map7D2, we sought to determine its functions 20 within the cell. To this end, we used N1-E115 cells that express Map7D2 and Map7D1 (Fig. 21 S2B and C). First, we analyzed the subcellular localization of Map7D2 in N1-E115 cells. 22 N1-E115 cells can undergo neuronal differentiation in response to DMSO under conditions of serum starvation (Kimhi, 1976), and most of the cells extend neurites up to $12 \mathrm{~h}$ after treatment with 1\% DMSO (Fig. S4A) (Smit, 2003). In both proliferating and differentiated cells, Map7D2 localized prominently to the centrosome and partially to MTs (Fig. 3A-C). These localizations were confirmed in N1-E115 cells stably expressing EGFP-rMap7D2 (Fig. 3D and E). Furthermore, during cytokinesis, Map7D2 accumulated at the midbody, where MT bundling occurs (Fig. 3B). Similarly, localization of Map7D2 was also observed at neurites, where MT bundling is also known to occur (Fig. 3C). Together with the biochemical properties, these subcellular localization data suggest that Map7D2 is involved in MT stabilization within the cell. 
$1 \quad$ Since N1-E115 cells express another Map7 family member, Map7D1, we also 2 determined its subcellular localization. Map7D1 exhibited similar localization to that of 3 Map7D2 in both proliferative and differentiated states (Fig. S4B-D). Interestingly, Map7D1

4 knock-down upregulated Map7D2 expression, as confirmed with three different siRNAs (Fig. 5 S2C), indicating that this effect was not due to the off-target effects of a particular siRNA. 6 Endogenous Map7D2 expression was suppressed in N1-E115 cells stably expressing

$7 \quad$ EGFP-rMap7D2 and was restored by specific knock-down of EGFP-rMap7D2 using $g f p$ siRNA 8 (Fig. 3D). These results suggest that the expression of Map7D2 was influenced by changes in 9 that of Map7D1. In contrast, Map7D2 knock-down did not affect Map7D1 expression (Fig. 10 S2C), and identical results were observed in the Map7d2 knock-out (Map7d2 ${ }^{-/}$) N1-E115 cells 11 we generated (Fig. S5A and B). As Map7D2 has the potential to functionally compensate for 12 Map7D1 loss, we decided to analyze the phenotypes of single and double knock-downs for 13 Map7D2 and Map7D1 in the following experiments.

Map7D2 is required for MT stabilization in the control of cell motility and neurite outgrowth.

17 It is well known that acetylation and detyrosination of $\alpha$-tubulins are associated with stable MTs 18 (Baas, 2016; Janke, 2017). Therefore, we examined the effects of Map7D2 or Map7D1 19 knock-down on the levels of acetylated and detyrosinated tubulins. Neither Map7D2 20 knock-down (Fig. 4A), nor Map7d2 knock-out affected the total levels of these modified 21 tubulins (Fig. 4B). In contrast, Map7D1 knock-down reduced the total level of acetylated but not detyrosinated tubulin (Fig. 4A), with double knock-down of Map7D2 and Map7D1 having the same impact (Fig. 4A). Consistently, immunostaining analysis also showed that Map7D1 knock-down greatly decreased the intensity of acetylated tubulin around the centrosome in N1-E115 cells (Fig. 4C and D). Map7D1 knock-down decreased the intensity of $\alpha$-tubulin and increased that of Map7D2 (Fig. 4C and D), indicating that Map7D1 is required for the maintenance of stable and acetylated MTs. Under Map7D2 knock-down, the intensity of $\alpha$-tubulin and Map7D1 decreased without affecting that of acetylated tubulin (Fig. 4C and D).

29 Together with our biochemical data for Map7D2, these results suggest that Map7D2 facilitates MT stabilization via direct binding, in contrast to Map7D1. 
1 Dysregulation of MT stabilization is known to affect various biological functions, for

2 instance, it can lead to increased cell motility and neurite outgrowth (Alesi, 2016; Biernat, 2002;

3 Grenningloh, 2004; Hubbert, 2002). Therefore, we analyzed whether random cell migration or

4 neurite outgrowth of N1-E115 cells is affected by single or double knock-down and Map7d2

$5 \quad$ knock-out. As expected, each single knock-down and the Map $7 d 2$ knock-out enhanced not only

6 the migration speed and distance during random cell migration (Fig. 5A-C), but also the rate of

7 neurite outgrowth (Fig. 5D). Furthermore, double knock-down of Map7D2 and Map7D1 tended

8 to result in increased cell motility and neurite outgrowth (Fig. 5B-D). Taken together, these

9 results suggest that Map7D2 and Map7D1 facilitate MT stabilization through distinct

10 mechanisms, thus controlling cell motility and neurite outgrowth.

\section{Discussion}

12 In the present study, we provide the first comprehensive analysis of Map7D2 biochemical

13 properties (Fig. 2). The N-terminal and C-terminal regions of Map7D2 exhibited high homology

14 to those of Map7 (Fig. S1). The N-terminal homologous region is basic and highly charged.

15 Most MT-binding domains characterized thus far are confined to positively charged regions

16 (Aizawa, 1990; Lewis, 1989; Noble, 1989; Pierre, 1992). Consistently, the MT-binding region

17 of Map7 was shown to be located at the N-terminal positively charged region (Masson, 1993).

18 Since we demonstrated that the N-terminal half of rMap7D2 directly bound to MTs (Fig. 2C), it

19 is likely that Map7D2 also associates with MTs through the positively charged N-terminal

20 region. Although the physiological role of the C-terminal region of Map7D2 is currently

21 unknown, it is conceivable that this region may play an important role as it is conserved

22 between Map7D2 and Map7. A region within the C-terminus of Map7 is required for complex

23 formation with Kif5b, the heavy chain of Kinesin-1 (Fig. S6), and is involved in

24 Kif5b-dependent transport by loading Kif5b onto MTs (Hooikaas, 2019; Kikuchi, 2018;

25 Metzger, 2012; Tymanskyj, 2018). Interestingly, we found that Map7D2 also formed a complex

26 with Kif5b (Fig. S6). Furthermore, it was recently reported that Map7D2 contributes to

27 Kinesin-1-mediated transport in the axons of hippocampal neurons (Pan, 2019). Taken together,

28 the biochemical properties are largely conserved between Map7D2 and Map7.

29 In contrast, the cellular functions of Map7D2 may differ from those of Map7. Our 30 group and Hooikaas et al. have previously reported that Map7 and Map7D1 have functional 31 overlaps in HeLa cells (Hooikaas, 2019; Kikuchi, 2018). For instance, both form a complex 
1 with Dishevelled, a mediator of Wnt5a signaling, while Map7D2 does not (Kikuchi, 2018). In 2 addition, Map7D2 exhibits distinct localization patterns in cultured hippocampal neurons, 3 localizing to the proximal axon (Pan, 2019). In the present study, we propose the molecular 4 mechanism how Map7D2 and Map7D1 regulate MT stabilization in N1-E115 cells (Fig. 5E). $5 \quad$ Map7D2 and Map7D1 both strongly localize to the centrosome and partially on MTs in 6 proliferating as well as in differentiated N1-E115 cells (Fig. 3 and Fig. S4B-D). Further, the 7 knock-down of either resulted in a comparable reduction of MT intensity (Fig. 4C and D), 8 enhancing the rate of cell motility and neurite outgrowth (Fig. 5A-D). Mechanistically, Map7D1 9 is required for the maintenance of MT acetylation, which is enriched in stable MTs, whereas 10 Map7D2 is not (Fig. 4). Taking these findings into consideration with our biochemical data, we 11 propose that, in contrast to Map7D1, Map7D2 facilitates stabilization by directly binding MTs 12 to then control cell motility and neurite outgrowth.

13 We also determined the tissue distribution of Map7D2, which has not been described 14 to date (Fig. 1). The tissue distribution of Map7 was previously analyzed using gene-trap mice 15 (Komada, 2000). At the mRNA level, Map7 is expressed in a variety of epithelial tissues, dorsal 16 root ganglia, trigeminal ganglia, and primitive seminiferous tubules during embryonic 17 development. We also reported that both Map7 and Map7D1 are expressed in the epithelia of 18 the mouse fallopian tube at the protein level (Kikuchi, 2018). Consistent with Map7 expression 19 in primitive seminiferous tubules, Map7 homozygous gene-trap mice exhibited defects in 20 spermatogenesis (Komada, 2000). Map7D2 was expressed predominantly in the glomerular 21 layer of the olfactory bulb and Sertoli cells of the testis (Fig. 1C). The glomerular layer is 22 known to be the region where axons accumulate and does not express Map2, a marker of 23 neuronal cell bodies and dendrites (Fig. 1C). As Map7D2 localizes to the proximal axon in 24 cultured hippocampal neurons (Pan, 2019), Map7D2 may have similar localization and function 25 in olfactory bulb neurons. The function of Map7D2 in Sertoli cells was not clarified in the 26 present study. Therefore, whether Map7D2 is involved in mammalian neurogenesis and 27 spermatogenesis represents a question for future research. 


\section{$1 \quad$ Materials and methods}

2 Molecular cloning, expression, and purification of rMap7D2

3 Based on the information of DDBJ/EMBL/GenBank accession number XM_228973, 4 oligonucleotide primers (5'-ATGTCGACATGGAGCGCAGCGGTGGGAACGGCG-3' and 5 5-ATGTCGACTCAACAGAAGGTGTTCAGCGTAGTTTC-3') were designed, and rat $6 \quad \operatorname{Map} 7 d 2(\mathrm{rMap} 7 d 2)$ cDNA was obtained by PCR using rat cDNA as a template. Expression 7 vectors for rMap7d2 were constructed in pCMV5-Myc (Nakanishi, 1997), pQE9 (Qiagen), 8 pGEX-5X-3 (Cytiva), pcDNA3.1/V5-His (Thermo Fisher Scientific), pCLXSN-GFP (Reiley, 9 2005), and pEGFP-N3 (Clontech). His 6 -tagged or GST-fused proteins were expressed in

10 Escherichia coli and purified using TALON metal affinity beads (CLONTECH) or 11 glutathione-Sepharose beads (Cytiva), respectively. GST-rMap7D2 (full length) was further 12 purified by gel filtration using a HiLoad 16/60 Superdex 200 column (Cytiva).

\section{Antibodies}

15 A rabbit polyclonal anti-Map7D2 antibody was raised against GST-rMap7D2 (aa1-235). All the 16 primary antibodies used are listed in Supplemental Table S1. Secondary antibodies coupled to 17 horseradish peroxidase (HRP) were purchased from Sigma-Aldrich. Alexa Fluor-conjugated 18 secondary antibodies used for immunofluorescence experiments were purchased from Thermo 19 Fisher Scientific.

\section{MT binding assay}

22 The MT co-sedimentation assay was performed as previously described, with a slight 23 modification (Yamamoto, 2002). MTs were prepared by incubating tubulin in polymerization 24 buffer (80 mM PIPES/NaOH, pH 6.8, $1 \mathrm{mM} \mathrm{MgCl}_{2}, 1 \mathrm{mM}$ EGTA, and $1 \mathrm{mM}$ GTP) containing $2510 \%$ glycerol for $20 \mathrm{~min}$ at $37^{\circ} \mathrm{C}$. After incubation, taxol was added at a final concentration of $2615 \mu \mathrm{M}$. Various amounts of rMap7D2 were incubated with $0.4 \mathrm{mg} / \mathrm{mL}$ of MTs in 27 polymerization buffer containing $15 \mu \mathrm{M}$ taxol for $20 \mathrm{~min}$ at $37^{\circ} \mathrm{C}$. After incubation with MTs, 28 the mixture $(200 \mu \mathrm{L})$ was placed over a $700-\mu \mathrm{L}$ cushion of $50 \%$ sucrose in polymerization 
1 buffer containing $15 \mu \mathrm{M}$ taxol. After the sample was centrifuged at $100,000 \times \mathrm{g}$ for $30 \mathrm{~min}$ at $237^{\circ} \mathrm{C}$, the supernatant was removed from the cushion, and the original volume was restored with 3 SDS sample buffer. Comparable amounts of the supernatant and pellet fractions were subjected 4 to SDS-PAGE, followed by CBB protein staining. The amount of protein was estimated using a 5 densitometer. ELISA for MT binding was performed in a 96-well microtiter plate as previously 6 described (Pedrotti, 1994). Briefly, wells were coated by incubating with $0.2 \mathrm{mg} / \mathrm{mL}$ of MTs in 7 polymerization buffer containing $15 \mu \mathrm{M}$ taxol for $2 \mathrm{~h}$ at $37^{\circ} \mathrm{C}$ and then blocked via incubation 8 with 5\% glycine. Increasing amounts of rMap7D2 were added to each well and incubated for 20 $9 \min$ at $37^{\circ} \mathrm{C}$. The plate was washed and further incubated with an anti-Map7D2 antibody. After 10 washing, the plates were incubated with a secondary antibody conjugated to horseradish 11 peroxidase. SuperSignal ELISA Pico (Pierce) was used as a chemiluminescent peroxidase 12 substrate.

\section{MT polymerization assays}

15 MT assembly was assayed by measuring turbidity at $350 \mathrm{~nm}$ using a spectrophotometer, as 16 previously described (Gaskin, 1974). Briefly, GST-rMap7D2 $(0.14 \mathrm{mg} / \mathrm{mL})$ was incubated with $172 \mathrm{mg} / \mathrm{mL}$ tubulin in polymerization buffer at $37^{\circ} \mathrm{C}$. The sample was continuously monitored at $18350 \mathrm{~nm}$ using a Hitachi U-2000 spectrophotometer. MT assembly was further assayed by 19 fluorescence microscopy using rhodamine-labeled tubulin (Hyman, 1991). Briefly, 20 GST-rMap7D2 $(0.07 \mathrm{mg} / \mathrm{ml})$ was incubated at $37^{\circ} \mathrm{C}$ for $20 \mathrm{~min}$ with $0.8 \mathrm{mg} / \mathrm{mL}$ tubulin $(1: 9=$ 21 rhodamine-labeled tubulin : unlabeled tubulin) in polymerization buffer. Incubation was stopped 22 through the addition of $1 \%$ glutaraldehyde. The sample was spotted onto a glass slide and 23 viewed under a fluorescence microscope.

\section{Northern blotting}

26 An RNA blot membrane (Clontech) was hybridized with the ${ }^{32} \mathrm{P}$-labeled full coding sequence of 27 rMap7D2, according to the manufacturer's protocol. 


\section{Cell culture and transfection}

$2 \mathrm{HeLa}$ and N1-E115 cells were cultured at $37^{\circ} \mathrm{C}$ in DMEM supplemented with $10 \%$ fetal bovine

3 serum and penicillin-streptomycin. The methods employed for plasmid or siRNA transfection

4 were previously described (Kikuchi, 2010). Plasmid transfection of N1-E115 cells was

5 performed using Lipofectamine LTX according to the manufacturer's instructions.

6 Differentiation of N1-E115 neuroblastoma cells was induced by decreasing the serum level to

$7 \quad 0.5 \%$ fetal bovine serum and adding $1 \%$ dimethyl sulfoxide (DMSO) (hereafter, the

8 above-described medium was referred to as differentiation medium). Stealth double-stranded

9 RNA was purchased from Thermo Fisher Scientific (USA). All siRNAs used in this study are

10 listed in Supplemental Table S2. Three individual siRNAs for mouse Map7d2 or Map7d1 were

11 designed based on the respective sequences. Double-stranded RNA targeting luciferase was

12 used as a control. The cells were cultured for $72 \mathrm{~h}$ and subjected to various experiments. In Fig.

13 4A, C, D, and Fig. 5B-D, to exclude siRNA off-target effects, a mixture of three individual

14 siRNAs for Map7D1 or Map7D2 was used. For the generation of N1-E115 cells stably 15 expressing EGFP-rMap7D2, clones were selected by adding G418 at $24 \mathrm{~h}$ post-transfection.

16 EGFP-rMap7D2 expression was confirmed by immunoblotting using antibodies against GFP 17 and Map7D2.

19 Generation of Map7d2 knock-out N1-E115 cell lines by CRISPR-Cas9

20 Two sgRNA sequences were designed using the CHOPCHOP CRISPR/Cas9 gRNA finder tool 21 (http://chopchop.cbu.uib.no/). The short double-stranded DNA for each sgRNA 22 (5-CACCGTGAAGAGAGCACATGTGCC-3 and 5-AAACGGCACATGTGCTCTCTTCAC-3, 23 or 5-CACCGCAGGATCACCAGGGCCTGG-3 and 24 5-AAACCCAGGCCCTGGTGATCCTGC-3') were inserted into the BbsI site of pX330 (Cong, 25 2013). To construct the Map7d2 knock-out vector, the $5^{\prime}$ and $3^{\prime}$ arms of each gene were 26 amplified by PCR using N1-E115 genomic DNA and cloned into the pCR4 Blunt-TOPO vector 27 (Thermo Fisher Scientific). The puromycin resistance marker was inserted between the 5' and 3' 28 arms (Fig. S6A). N1-E115 cells were transfected with $1 \mu \mathrm{g}$ of each of the two pX330-sgRNA 29 plasmids and the knock-out vector using Lipofectamine LTX (Thermo Fisher Scientific). 30 Knock-out clones were selected by adding puromycin (Sigma-Aldrich) at $24 \mathrm{~h}$ post-transfection. 
1 Successful knock-out was confirmed by immunoblotting using an anti-Map7D2 antibody and 2 genomic PCR.

\section{$4 \quad$ Animals}

5 Mice (C57BL6/N; Japan SLC, Japan) were used in this study. Animal care and experiments 6 were conducted in accordance with the guidelines for the care and use of laboratory animals of 7 the Center for Animal Resources and Development, Kumamoto University. All experiments 8 were approved by the experimental animal ethics committee of Kumamoto University 9 (A2019-127 and A2021-018). Mice were kept in a light- and temperature-controlled room with 10 a 12 -h light/dark cycle at $22 \pm 1{ }^{\circ} \mathrm{C}$.

\section{Quantitative real-time PCR}

13 Each RNA sample was subjected to reverse transcription using murine leukemia virus reverse 14 transcriptase (Thermo Fisher Scientific), and the generated cDNA was used as a template for 15 qRT-PCR. Each reaction mixture was prepared using the KAPA SYBR Fast qPCR kit (Kapa 16 Biosystems), and the PCR reaction was performed on ViiA7 (Thermo Fisher Scientific). The 17 primers used for RT-qPCR are listed in Supplementary Table S3.

\section{Immunoblotting and immunoprecipitation}

20 For immunoblotting, cells were washed once with PBS and lysed with Laemmli's sample buffer.

21 After boiling, the lysates were separated by SDS-PAGE, transferred to PVDF membranes 22 (Millipore), and immunoblotted with antibodies. For immunoprecipitation analysis, the HeLa 23 cells were washed once with PBS at $24 \mathrm{~h}$ post-transfection and lysed with $1 \times$ NP40 buffer [20 $24 \mathrm{mM}$ Tris- $\mathrm{HCl}$ (pH 8.0), $10 \%$ glycerol, $137 \mathrm{mM} \mathrm{NaCl}, 1 \%$ NP40] supplemented with protease 25 inhibitors and phosphatase inhibitors for $20 \mathrm{~min}$ on ice. The supernatant was collected after 26 centrifugation and incubated with the appropriate antibodies. After incubation, $15 \mu \mathrm{L}$ of protein 27 A or G Sepharose beads was added, and the mixtures were rotated for $1 \mathrm{~h}$ at $4^{\circ} \mathrm{C}$. The beads 
1 were washed once with $1 \times \mathrm{NP} 40$ buffer, twice with $\mathrm{LiCl}$ buffer [0.1 M Tris-HCl (pH 7.5), $0.5 \mathrm{M}$

$2 \mathrm{LiCl}$, once with $10 \mathrm{mM}$ Tris- $\mathrm{HCl}$ ( $\mathrm{pH} 7.5)$, and were finally resuspended in Laemmli's sample 3 buffer.

\section{$5 \quad$ Immunofluorescence staining}

6 For immunofluorescence staining, cells were grown on coverslips and fixed in $100 \%$ methanol 7 at $-20{ }^{\circ} \mathrm{C}$ for $5 \mathrm{~min}$. After blocking with $1 \%$ BSA in PBS for $1 \mathrm{~h}$ at room temperature, the 8 samples were incubated with primary antibodies overnight at $4{ }^{\circ} \mathrm{C}$, followed by incubation with 9 Alexa Fluor-conjugated secondary antibodies (Thermo Fisher Scientific) for $1 \mathrm{~h}$. For 10 immunofluorescence tissue staining, tissues were fixed in $4 \%$ paraformaldehyde in PBS at $4{ }^{\circ} \mathrm{C}$ 11 overnight, and then immersed sequentially in 10, 20, and $30 \%$ sucrose in PBS at $4^{\circ} \mathrm{C}$. After 12 sucrose equilibration, tissues were immersed in OCT (Sakura Finetechnical) at room 13 temperature for $5 \mathrm{~min}$, followed by embedding in OCT and freezing in liquid nitrogen. Sections $14(10 \mu \mathrm{m})$ were stored at $-80^{\circ} \mathrm{C}$. The sections were washed once with PBS for $10 \mathrm{~min}$ and twice 15 with $0.1 \%$ Triton X-100 in PBS for 10 min. After blocking with Blocking One (Nacalai) for $1 \mathrm{~h}$ 16 at room temperature, the samples were incubated with primary antibodies overnight at $4{ }^{\circ} \mathrm{C}$, 17 followed by incubation with Alexa Fluor-conjugated secondary antibodies (Thermo Fisher 18 Scientific) for $1 \mathrm{~h}$. Nuclei were stained with DAPI for $30 \mathrm{~min}$ at room temperature. The samples 19 were viewed under a fluorescence microscope (Olympus, BX51) or a confocal microscope 20 (Olympus, FV1000 or Leica, TCS SP8). Images were processed and analyzed using Fiji 21 software (National Institutes of Health).

\section{Random cell migration assay and neurite outgrowth assay}

24 For the random cell migration assay, cells were seeded onto a laminin-coated $(10 \mu \mathrm{g} / \mathrm{mL})$ 25 glass-bottom dish and recorded under an inverted microscope system equipped with an 26 incubator (Olympus, LCV110). For the neurite outgrowth assay (Fig. S5C), the underside of 3 $27 \mu \mathrm{m}$ pore transwell membranes (Corning) was coated with $500 \mu \mathrm{L}$ of $10 \mu \mathrm{g} / \mathrm{mL}$ laminin in PBS 28 into a well of a 24-well plate. After coating, the membranes were removed from the laminin and 29 placed into the well of a 24 -well dish containing $500 \mu \mathrm{L}$ differentiation media. One hundred 
1 microliters of cell suspension (containing $1-2 \times 10^{5}$ cells) was added to the insert chamber on

2 top of the membrane. The cells were allowed to extend neurites through the membrane pores to

3 the lower chamber (underside of the membrane) for $6 \mathrm{~h}$ at $37^{\circ} \mathrm{C}$. The cells were then fixed and

4 stained with an anti- $\alpha$-tubulin antibody. Images were processed and analyzed using Fiji software

5 (National Institutes of Health).

\section{$7 \quad$ Statistics}

8 The experiments were performed at least three times (biological replicates), and the results are

9 expressed as the average \pm S.D. or the median, first and third quartiles, and 5-95\% confidence

10 intervals for the box-and-whisker plot. Differences between data values were tested for

11 statistical significance using the Student's $t$-test. Statistical significance was set at $P<0.05$.

12

\section{Other Procedures}

14 Tubulin was prepared from fresh porcine brains by three cycles of polymerization and

15 depolymerization, followed by DEAE-Sephadex column chromatography (Shelanski, 1973;

16 Williams R.C.Jr. and Lee, 1982). 


\section{Acknowledgements}

2 We thank past and present members of our laboratory for helpful discussions. This work was

3 partly carried out at the Institute of Molecular Embryology and Genetics, the Gene Technology

4 Centre, the Center for Animal resources and Development, and Research Facilities of the

5 School of Medicine, Kumamoto University.

\section{Author Contributions}

8 K.K. designed the research and carried out most of the experiments, except for Fig. 1A, B, and 2.

9 Y.S., A.U., H.Y., and H.N. carried out Fig. 1A, B and 2, and raised the rabbit polyclonal 10 anti-Map7D2 antibody. K.I. provided technical assistance for the immunofluorescence analysis 11 using the mouse testis. K.S. provided technical assistance for the immunofluorescence analysis 12 using the mouse brain. Y.S., T.S., S.H., and H.N. provided reagents, materials, and analysis 13 tools. K.K. wrote the paper, and Y.S., and H.N. edited the paper. All of the authors discussed 14 the results and commented on the manuscript.

\section{Funding}

17 This work was supported by JSPS KAKENHI Grant Numbers 22700881, 24700980, 15K07054, 18 and 19K06664, and grants from The Japan Spina Bifida \& Hydrocephalus Research Foundation, 19 Astellas Foundation for Research on Metabolic Disorders, the Mochida Memorial Foundation, 20 the Takeda Science Foundation, and the Uehara Memorial Foundation (to K.K.).

\section{Conflict of Interest}

23 The authors declare that the research was conducted in the absence of any commercial or 24 financial relationships that could be construed as a potential conflict of interest. 


\section{References}

Aizawa, H., Emori, Y., Muroushi, H., Kawasaki, H., Sakai, H., and Suzuki, K. 1990. Molecular cloning of a ubiquitously distributed microtubule-associated protein with Mr 190,000. J. Biol. Chem. 265:13849-13855.

Alesi, G.N., Jin, L., Li, D., Magliocca, K.R., Kang, Y., and Chen, Z.G. 2016. RSK2 signals through stathmin to promote microtubule dynamics and tumor metastasis. Oncogene. 35:5412-5421.

Baas, P.W., Rao, A.N., Matamoros, A.J., and Leo, L. 2016. Stability properties of neuronal microtubules. Cytoskeleton (Hoboken). 73:442-460.

Barlan, K., Lu, W., and Gelfand, V.I. 2013. The microtubule-binding protein ensconsin is an essential cofactor of kinesin-1. Curr. Biol. 23:317-322.

Biernat, J., Wu, Y.Z., Timm, T., Zheng-Fischhöfer, Q., Mandelkow, E., Meijer, L., and Mandelkow, E.M. 2002. Protein Kinase MARK/PAR-1 Is Required for Neurite Outgrowth and Establishment of Neuronal Polarity. Mol. Biol. Cell. 13:4013-4028.

Bulinski, J.C., and Bossler, A. 1994. Purification and characterization of ensconsin, a novel microtubule stabilizing protein. J. Cell Sci. 107:2839-2849.

Carles, G., Braguer, D., Dumontet, C., Bourgarel, V., Goncalves, A., Sarrazin, M., Rognoni, J.B., Briand, C. 1999. Differentiation of human colon cancer cells changes the expression of $\beta$-tubulin isotypes and MAPs. Br. J. Cancer. 80:1162-1168.

Cleary, J.M., and Hancock, W.O. 2021. Molecular mechanisms underlying microtubule growth dynamics. Curr. Biol. 31:R560-R573.

Cong, L., Ran, F.A., Cox, D., Lin, S., Barretto, R., Habib, N., Hsu, P.D., Wu, X., Jiang, W., Marraffini, L.A., and Zhang, F. 2013. Multiplex genome engineering using CRISPR/Cas systems. Science. 339:819-823.

Etienne-Manneville, S. 2013. Microtubules in cell migration. Annu. Rev. Cell Dev. Biol. 29:471-499.

Fabre-Jonca, N., Viard, I., French, L.E., and Masson, D. 1999. Upregulation and Redistribution of E-MAP-115 (Epithelial Microtubule-Associated Protein of 115 $\mathrm{kDa}$ ) in Terminally Differentiating Keratinocytes is Coincident with the Formation of Intercellular Contacts. J. Invest. Dermatol. 112:216-225. 
1 Gaskin, F., Cantor, C. R., and Shelanski, M. L. 1974. Turbidimetric studies of the in vitro assembly and disassembly of porcine neurotubules. J. Mol. Biol. 89:737-758.

4 Grenningloh, G., Soehrman, S., Bondallaz, P., Ruchti, E., and Cadas, H. 2004. Role of the microtubule destabilizing proteins SCG10 and stathmin in neuronal growth. J. Neurobiol. 58:60-69.

7 Gustke, N., Trinczek, B., Biernat, J., Mandelkow, E.M., and Mandelkow, E. 1994. Domains of tau Protein and Interactions with Microtubules. Biochemistry 33:9511-9522.

Hooikaas, P.J., Martin, M., Mühlethaler, T., Kuijntjes, G.J., Peeters, C.A.E., Katrukha, E.A., Ferrari, L., Stucchi, R., Verhagen, D.G.F., van Riel, W.E., Grigoriev, I., Altelaar, A.F.M., Hoogenraad, C.C., Rüdiger, S.G.D., Steinmetz, M.O., Kapitein, L.C., and Akhmanova, A. 2019. MAP7 family proteins regulate kinesin-1 recruitment and activation. J. Cell Biol. 218:1298-1318.

Hubbert, C., Guardiola, A., Shao, R., Kawaguchi, Y., Ito, A., Nixon, A., Yoshida, M., Wang, X.F., and Yao, T.P. 2002. HDAC6 is a microtubule-associated deacetylase. Nature. 417:455-458.

Hyman, A., Drechsel, D., Kellogg, D., Salser, S., Sawin, K., Steffen, P., Wordeman, L., and Mitchison, T. 1991. Preparation of modified tubulins. Methods Enzymol. 196:478-485.

Janke, C., and Montagnac, G. 2017. Causes and Consequences of Microtubule Acetylation. Curr. Biol. 27:R1287-R1292.

Kikuchi, K., Nakamura, A., Arata, M., Shi, D., Nakagawa, M., Tanaka, T., Uemura, T., Fujimori, T., Kikuchi, A., Uezu, A., Sakamoto, Y., and Nakanishi, H. 2018. Map7/7D1 and Dvl form a feedback loop that facilitates microtubule remodeling and Wnt5a signaling. EMBO Rep. 19:e45471.

Kikuchi, K., Niikura, Y., Kitagawa, K., and Kikuchi, A. 2010. Dishevelled, a Wnt signalling component, is involved in mitotic progression in cooperation with Plk1. EMBO J. 29:3470-3483.

Kimhi, Y., Palfrey, C., Spector, I., Barak, Y., and Littauer, U.Z. 1976. Maturation of neuroblastoma cells in the presence of dimethylsulfoxide. Proc. Natl. Acad. Sci. U. S. A. 73:462-466.

Komada, M., McLean, D.J., Griswold, M.D., Russelll, L.D., and Soriano, P. . 2000. E-MAP-115, encoding a microtubule-associated protein, is a retinoic acid-inducible gene required for spermatogenesis. Genes Dev. 14:1332-1342. 
1 Lansbergen, G., Komarova, Y., Modesti, M., Wyman, C., Hoogenraad, C.C., Goodson, 2 H.V., Lemaitre, R.P., Drechsel, D.N., van Munster, E., Gadella Jr.T.W., 3 Grosveld, F., Galiart, N., Borisy, G.G., and Akhmanova, A. 2004. 4 Conformational changes in CLIP-170 regulate its binding to microtubules and $5 \quad$ dynactin localization. J. Cell Biol. 166:1003-1014.

6 Lewis, S.A., Ivanov, I.E., Lee, G.H., and Cowan, N.J. 1989. Organization of 7 microtubules in dendrites and axons is determined by a short hydrophobic zipper

Masson, D., and Kreis, T. E. 1993. Identification and molecular characterization of E-MAP-115, a novel microtubule-associated protein predominantly expressed in epithelial cells. J. Cell Biol. . 123:357-371.

Metzger, T., Gache, V., Xu, M., Cadot, B., Folker, E.S., Richardson, B.E., Gomes, E.R., and Baylies, M.K. 2012. MAP and kinesin-dependent nuclear positioning is required for skeletal muscle function. Nature. 484:120-124.

Monroy, B.Y., Sawyer, D.L., Ackermann, B.E., Borden, M.M., Tan, T.C., and Ori-McKenney, K.M. 2018. Competition between microtubule-associated proteins directs motor transport. Nat. Commun. 9:1487.

Monroy, B.Y., Tan, T.C., Oclaman, J.M., Han, J.S., Simó, S., Niwa, S., Nowakowski, D.W., McKenney, R.J., and Ori-McKenney, K.M. 2020. A Combinatorial MAP Code Dictates Polarized Microtubule Transport. Dev. Cell. 53:60-72.e64.

Nakanishi, H., Obaishi, H., Satoh, A., Wada, M., Mandai, K., Satoh, K., Nishioka, H., Matsuura, Y., Mizoguchi, A., and Takai, Y. 1997. Neurabin: a novel neural tissue-specific actin filament-binding protein involved in neurite formation. $J$. Cell Biol. 139:951-961.

Noble, M., Lewis, S.A., and Cowan, N.J. 1989. The microtubule binding domain of microtubule-associated protein MAP1B contains a repeated sequence motif unrelated to that of MAP2 and tau. J. Cell Biol. 109:3367-3376.

Pan, X., Cao, Y., Stucchi, R., Hooikaas, P. J., Portegies, S., Will, L., Martin, M., Akhmanova, A., Harterink, M., and Hoogenraad, C. C. 2019. MAP7D2 Localizes to the Proximal Axon and Locally Promotes Kinesin-1-Mediated Cargo Transport into the Axon. Cell Rep. 26:1988-1999.

Pedrotti, B., Colombo, R., and Islam, K. 1994. Interactions of microtubule-associated protein MAP2 with unpolymerized and polymerized tubulin and actin using a 96-well microtiter plate solid-phase immunoassay. Biochemistry. 33:8798-8806. 
1 Pierre, P., Scheel, J., Richard, J.E., and Kreis, T.E. 1992. CLIP-170 links endocytic vesicles to microtubules. Cell. 70:887-900.

3 Reiley, W., Zhang, M., Wu, X., Granger, E., and Sun, S.C. 2005. Regulation of the 4 deubiquitinating enzyme CYLD by IkappaB kinase gamma-dependent phosphorylation. Mol. Cell. Biol. 25:3886-3895.

Sakamoto, T., Uezu, A., Kawauchi, S., Kuramoto, T., Makino, K., Umeda, K., Araki, N.,

\section{Figures} Baba, H., and Nakanishi, H. . 2008. Mass spectrometric analysis of microtubule co-sedimented proteins from rat brain. Genes Cells. 13:295-312.

Shelanski, M.L., Gaskin, F., and Cantor, C. R. 1973. Microtubule Assembly in the Absence of Added Nucleotides. Proc. Natl. Acad. Sci. USA 70:765-768.

Smit, M., Leng, J., and Klemke, R.L. 2003. Assay for neurite outgrowth quantification. BioTechniques. 35:254-256.

Sung, H.H., Telley, I.A., Papadaki, P., Ephrussi, A., Surrey, T., and Rørth, P. 2008. Drosophila ensconsin promotes productive recruitment of Kinesin-1 to microtubules. Dev. Cell. 15:866-876.

Tymanskyj, S.R., Yang, B.H., Verhey, K.J., and Ma, L. 2018. MAP7 regulates axon morphogenesis by recruiting kinesin-1 to microtubules and modulating organelle transport. Elife. 7:e36374.

Williams R.C.Jr. and Lee, J.C. 1982. Preparation of tubulin from brain. Methods Enzymol. 85:376-385.

Yadav, S., P.J. Verma, and D. Panda. 2014. C-terminal region of MAP7 domain containing protein 3 (MAP7D3) promotes microtubule polymerization by binding at the C-terminal tail of tubulin. PLoS One. 9:e99539.

Yamamoto, H., Yamauchi, E., Taniguchi, H., Ono, T., and Miyamoto, E. 2002. Phosphorylation of microtubule-associated protein tau by $\mathrm{Ca} 2+/$ calmodulin-dependent protein kinase II in its tubulin binding sites. Arch. Biochem. Biophys. 408:255-262.

\section{Figure 1. Tissue distribution of Map7D2.}


1 (A) Northern blot analysis. An RNA blot membrane (CLONTECH) was hybridized with the

$2 \quad{ }^{32} \mathrm{P}$-labeled full coding sequences of rMap7D2 according to the manufacturer's protocol. (B)

3 Immunoblotting analysis. Various tissue lysates (20 $\mu \mathrm{g}$ of protein) were subjected to

4 SDS-PAGE, followed by immunoblotting with the anti-Map7D2 antibody. (C) Expression

5 patterns of Map7D2 in the brain and testis by immunofluorescence. Upper panels, frozen

6 sagittal sections of postnatal day 0 mouse brains were stained with anti-Map7D2 (magenta) and

7 antibodies against mature neuron marker Map2 (green). DNA was labeled with DAPI (gray).

8 For a comparison of signal intensities, images were captured under the same parameters.

9 Contrast-enhanced images of Map7D2 staining were shown in the rightmost column. Lower

10 panels, frozen coronal sections of adult mouse testis were stained with anti-Map7D2 (magenta)

11 and antibodies against Sertoli cell marker Tubb3 (green). DNA was labeled with DAPI (gray).

12 Scale bars in upper panels or lower panels: 100 or $50 \mu \mathrm{m}$, respectively.

14 Figure 2. Map7D2 has the ability to stabilize MTs.

15 (A) Schematic structures of hMap7, hMap7D2, and rMap7D2. (B) Co-sedimentation of 16 rMap7D2 with MTs. Left panel, His 6 -rMap7D2 $(34 \mu \mathrm{g} / \mathrm{mL})$ was mixed with MTs, followed by 17 ultracentrifugation. Comparable amounts of the supernatant and pellet fractions were subjected 18 to SDS-PAGE, followed by CBB protein staining. S, supernatant; P, pellet. Middle panel, 19 various amounts of His $_{6}$-rMap7D2 were mixed with MTs, followed by ultracentrifugation. 20 Amounts of free and bound $\mathrm{His}_{6}$-rMap7D2 were calculated by determining protein amounts 21 from the supernatant and pellet fractions, respectively, with a densitometer. Right panel, 22 Scatchard analysis. (C) Location of the MT-binding domain. GST-rMap7D2-N (80 $\mu \mathrm{g} / \mathrm{mL})$ or 23 GST-rMap7D2-C $(200 \mu \mathrm{g} / \mathrm{mL})$ was mixed with MTs, followed by ultracentrifugation. 24 Comparable amounts of the supernatant and pellet fractions were subjected to SDS-PAGE, 25 followed by CBB protein staining. S, supernatant; and P, pellet. (D) Turbidity measurement. 26 GST-rMap7D2 was mixed with tubulin. The sample was incubated at $37^{\circ} \mathrm{C}$ and continuously 27 monitored at $350 \mathrm{~nm}$ using a spectrophotometer. (०) without GST-rMap7D2; and $(\bullet)$ with 28 GST-rMap7D2. *, $P<0.003$ (the F-test). (E) Immunofluorescent observation. GST-rMap7D2 29 was incubated for $20 \mathrm{~min}$ at $37^{\circ} \mathrm{C}$ with rhodamine-labeled tubulin. After fixation, the sample 30 was spotted on a slide glass and viewed under a fluorescence microscope. (F) HeLa cells 31 transiently overexpressing Myc-rMap7D2. Myc-rMap7D2 was transfected into HeLa cells, and 
1 the cells were then double-stained with anti-Myc and anti- $\alpha$-tubulin antibodies. Arrowheads

2 show MT bundles. Scale bars, $50 \mu \mathrm{m}$ in $\mathrm{E}$ and $10 \mu \mathrm{m}$ in F.

4 Figure 3. Subcellular localization of Map7D2 in proliferative and differentiated N1-E115 5 cells.

6 (A-C) Localization of Map7D2 in interphase (A), mitosis $(\mathbf{B})$, and differentiation state (C) of 7 N1-E115 cells. Cells were double-stained with anti-Map7D2 and anti- $\alpha$-tubulin antibodies. In A, 8 the insets show enlarged images of regions indicated by a white box. In $\mathbf{B}$, the inset shows 9 metaphase cells. In $\mathbf{C}$, images of differentiated cells were captured by z-sectioning, and each 10 inset show enlarged images of regions indicated by a white box at each focal plane. Arrowheads 11 show the centrosomal localization of Map7D2. (D) Generation of N1-E115 cells stably 12 expressing EGFP-rMap7D2. To check the expression level of EGFP-rMap7D2, lysates derived 13 from the indicated cells were probed with anti-GFP (top panel) and anti-Map7D2 (middle panel) 14 antibodies. The blot was reprobed for $\gamma$-tubulin as a loading control (bottom panel). Of note, 15 stable expression of EGFP-rMap7D2 reduced endogenous Map7D2 expression, and specific 16 knock-down of EGFP-rMap7D2 using $g f p$ siRNA restored endogenous Map7D2 expression. (E) 17 Confirmation for subcellular localization of Map7D2 using N1-E115 cells stably expressing 18 EGFP-rMap7D2. Images were captured by z-sectioning. Top panels show images taken with the 19 lower or upper focal plane, and bottom panels show the image reconstructed in the $\mathrm{z}$-axis 20 direction. Arrow head shows centrosomal localization of Map7D2. Scale bars: $10 \mu \mathrm{m}$.

Figure 4. Map7D2 is required for MT stabilization within the cell.

23 (A) Immunoblot analysis for acetylated (Ace-) and detyrosinated (Detyr-) tubulin in cells treated 24 with each siRNA. Lysates derived from the indicated cells were separated by SDS-PAGE and 25 subjected to immunoblotting with anti-Map7D1, anti-Map7D2, anti-Ace-tubulin, or 26 anti-Detyr-tubulin antibodies. The blot was reprobed for Clathrin heavy chain (HC) or $\alpha$-tubulin 27 as a loading control. (B) Immunoblot analysis for Ace- and Detyr-tubulins in wild-type and $28 \operatorname{Map} \mathrm{d} 2^{-/}$cells. Three independent $\operatorname{Map} 7 d 2^{-/}$clones were used in this study. Lysates derived 29 from the indicated cells were separated by SDS-PAGE and were immunoblotted with 
1 anti-Map7D1, anti-Map7D2, anti-Ace-tubulin, or anti-Detyr-tubulin antibodies. The blot was

2 reprobed for $\alpha$-actin or $\alpha$-tubulin as a loading control. (C) Immunofluorescence staining for

$3 \alpha$-tubulin, Ace-tubulin, and Map7D1 or Map7D2 in cells treated with each siRNA. For a

4 comparison of signal intensities, images were captured under the same parameters. The insets

5 show enlarged images of regions indicated by a white box. Of note, Ace-tubulin was present

6 predominately around the centrosome in N1-E115 cells. (D) Quantification for

7 immunofluorescence staining shown in C. Left panels, the intensities of $\alpha$-tubulin, Ace-tubulin,

8 and Map7D1 around the centrosome in the indicated cells were measured via ROI analysis

9 (control, $\mathrm{n}=197$ cells; siMap7d2, $\mathrm{n}=192$ cells from three independent experiments). Right

10 panels, the intensities of $\alpha$-tubulin, Ace-tubulin, and Map7D2 around the centrosome in the

11 indicated cells were measured by ROI analysis (control, $\mathrm{n}=193$ cells; siMap7d1, $\mathrm{n}=227$ cells

12 from three independent experiments). *, $P<1 \times 10^{-13} ; * *, P<1 \times 10^{-8}$ (the Student's $t$-test). Scale

13 bars: $10 \mu \mathrm{m}$ in $\mathrm{C}$ and $5 \mu \mathrm{m}$ in $\mathrm{D}$.

\section{Figure 5. Map7D2 suppresses random cell migration and neurite outgrowth.}

16 (A) Bright-field images of migrating N1-E115 cells. Arrowheads show lamellipodia formed in 17 the direction of migration. (B) Tracking analysis of random cell migration in the indicated cells.

18 Each color represents the trajectory of 12 randomly selected cells. (C) Velocity and net distance 19 measured in the indicated cells (control: $\mathrm{n}=114$ cells; siMap7d1: $\mathrm{n}=100$ cells; siMap7d2: $\mathrm{n}=$ 2071 cells; siMap7d1/d2: $\mathrm{n}=107$ cells; Map7d2 $2^{-\alpha} \mathrm{n}=60$ cells from three independent 21 experiments). *, $P<1 \times 10^{-4}$; **, $P<0.002$ (the Student's $t$-test). (D) Neurite outgrowth assay in the 22 indicated cells. Neurites and cell bodies were visualized by $\alpha$-tubulin staining (upper). The 23 neurite outgrowth from each cell was distinguished by acquiring images with Z-sectioning. Data 24 are from three or four independent experiments and represent the average $\square \pm \square$ S.D. (lower). *, $25 P<0.002$; **, $P<0.0002$ (the Student's $t$-test). (E) Proposed model for the distinct mechanisms of 26 Map7D2 and Map7D1 for MT stabilization. See Discussion for further detail. Scale bars in A 27 and D: $20 \mu \mathrm{m}$. 
A

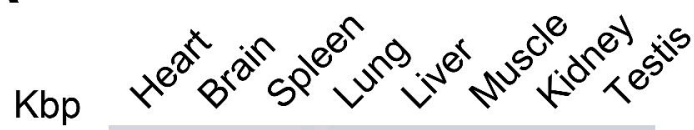
9.5-

$7.5^{-}$

4.4-

2.4-

1.35 -

$0.24-$

C

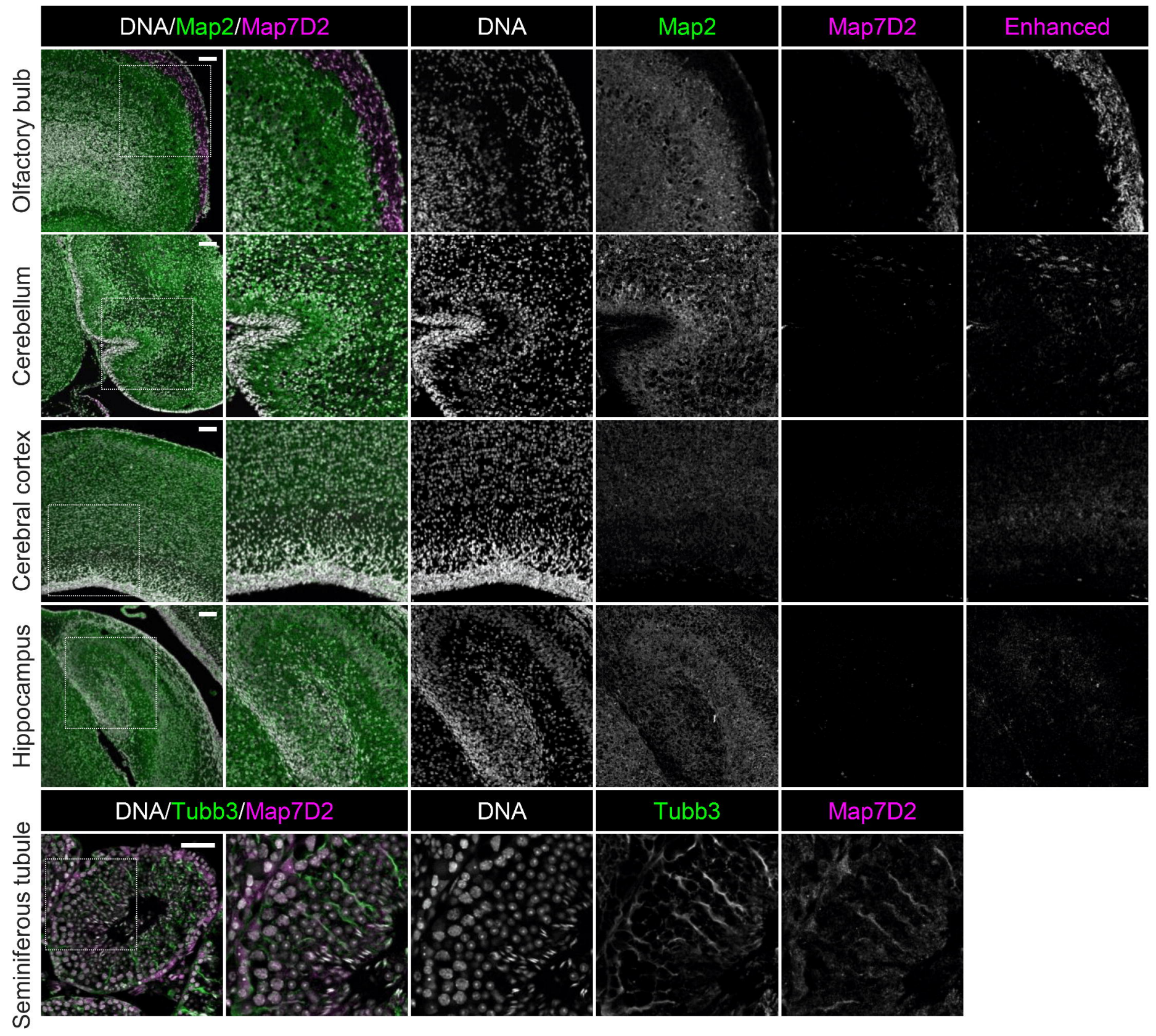

B

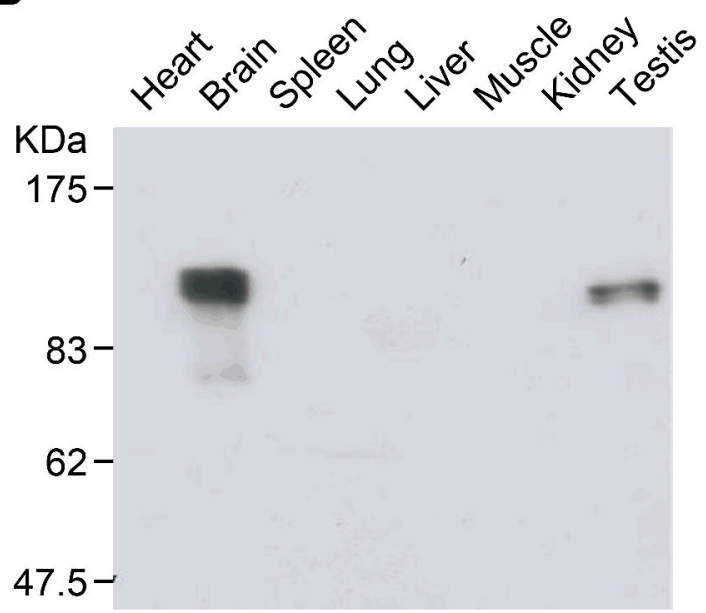




\section{Kikuchi_Figure 2}

A

hMap7

\begin{tabular}{|llll|}
\hline 87 & 172 & 490 & 641
\end{tabular} 749 aa $\quad$ Core conserved region

hMap7D2

rMap7D2

\begin{tabular}{|c|c|c|c|c|}
\hline 53 & 138 & 389 & 562 & \multirow{2}{*}{ Identity: $68.1 \%$} \\
\hline 57 & 142 & 422 & 593 & \\
\hline
\end{tabular}

rMap7D2-N

12

rMap7D2-C in Fig. 3C

B

$\mathrm{KDa}$

116 -

$97-$

$66-$
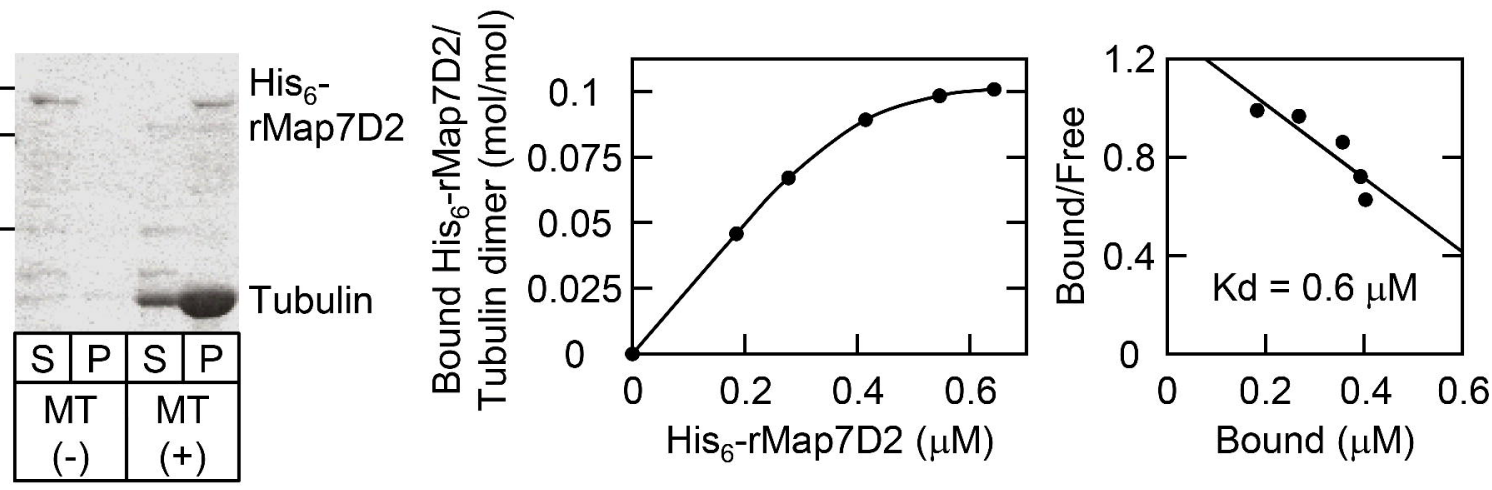

C

$\mathrm{KDa}$

97-

66-

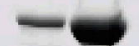

-

$-6$

45

\begin{tabular}{|c|c|c|c|c|c|c|c|}
\hline S & P & S & P & S & P & S & P \\
\hline MT & MT & MT & MT \\
$(-)$ & $(+)$ & $(-)$ & $(+)$ \\
\hline \multicolumn{3}{|c|}{ GST- } & \multicolumn{3}{|c|}{ GST- } \\
rMap7D2-N & rMap7D2-C \\
\hline
\end{tabular}

D

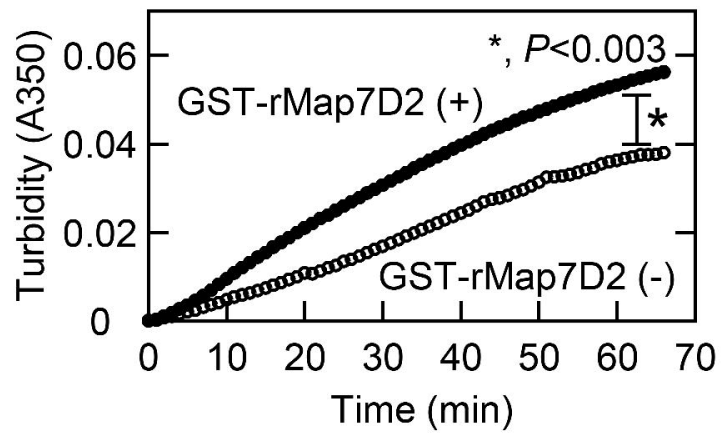

E

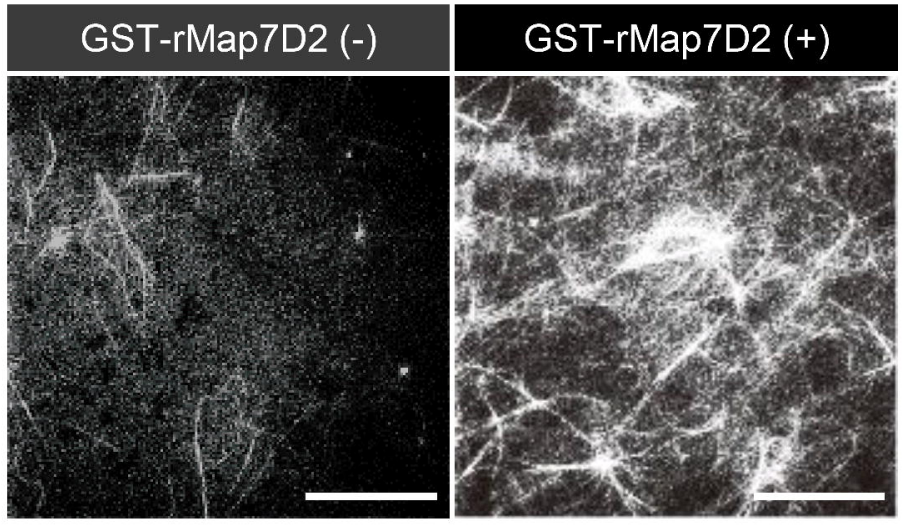

F

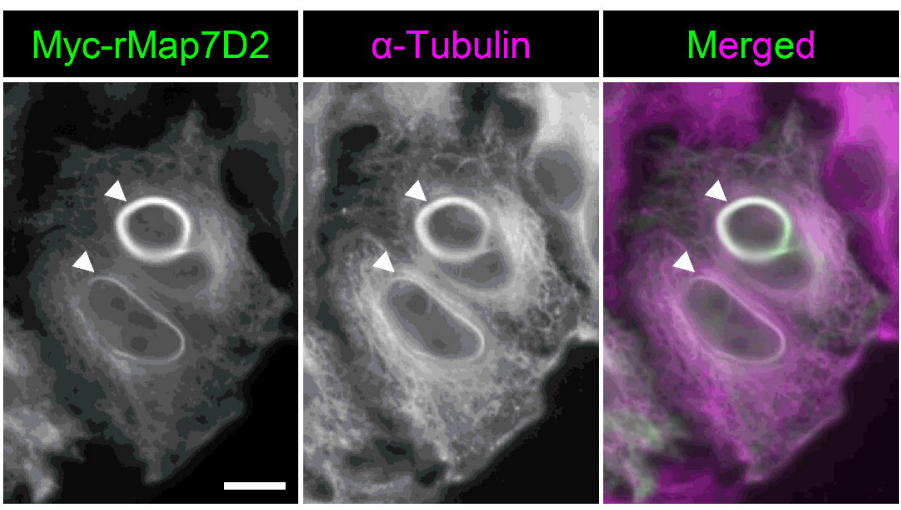


Kikuchi_Figure 3

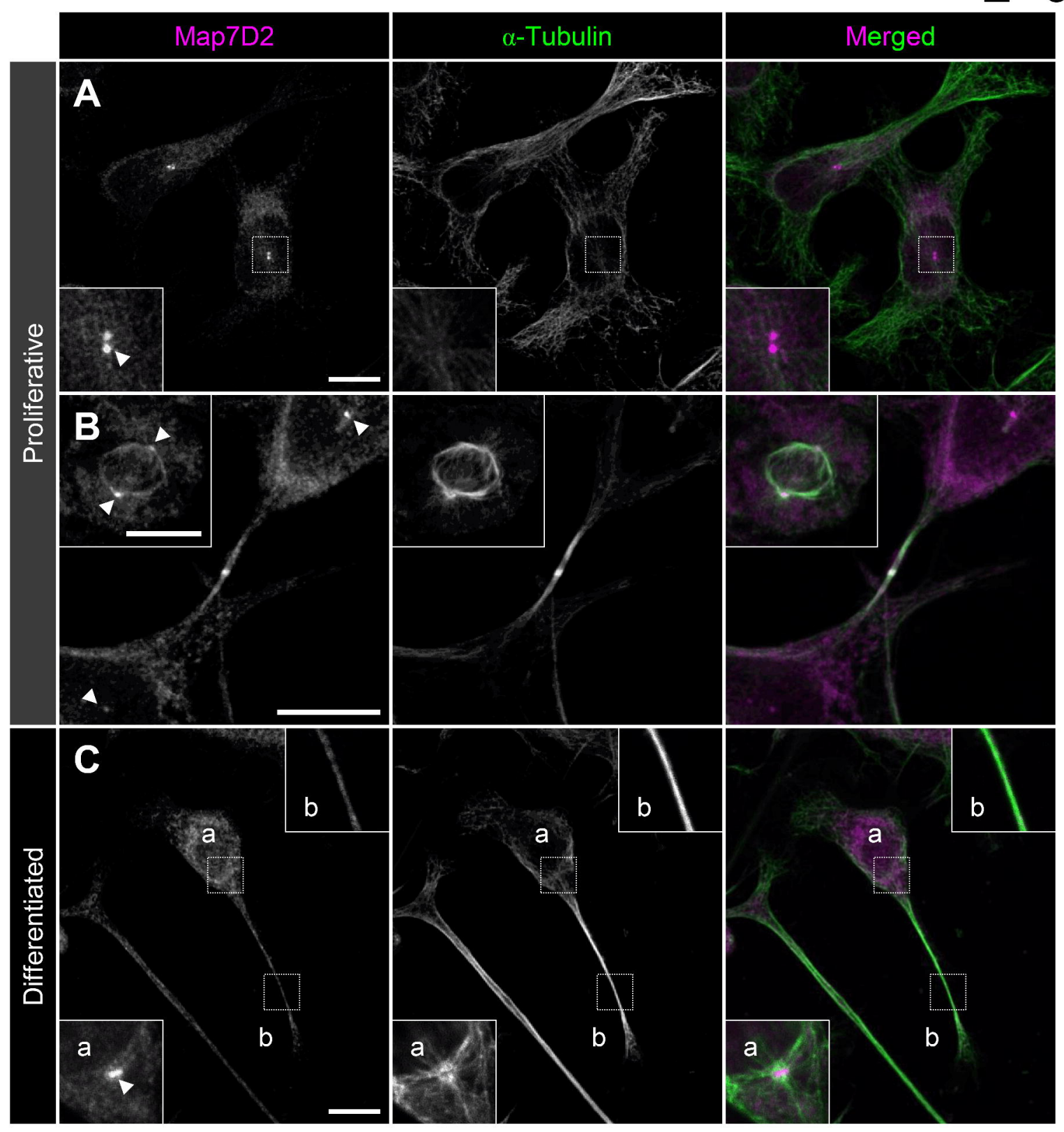

D

E

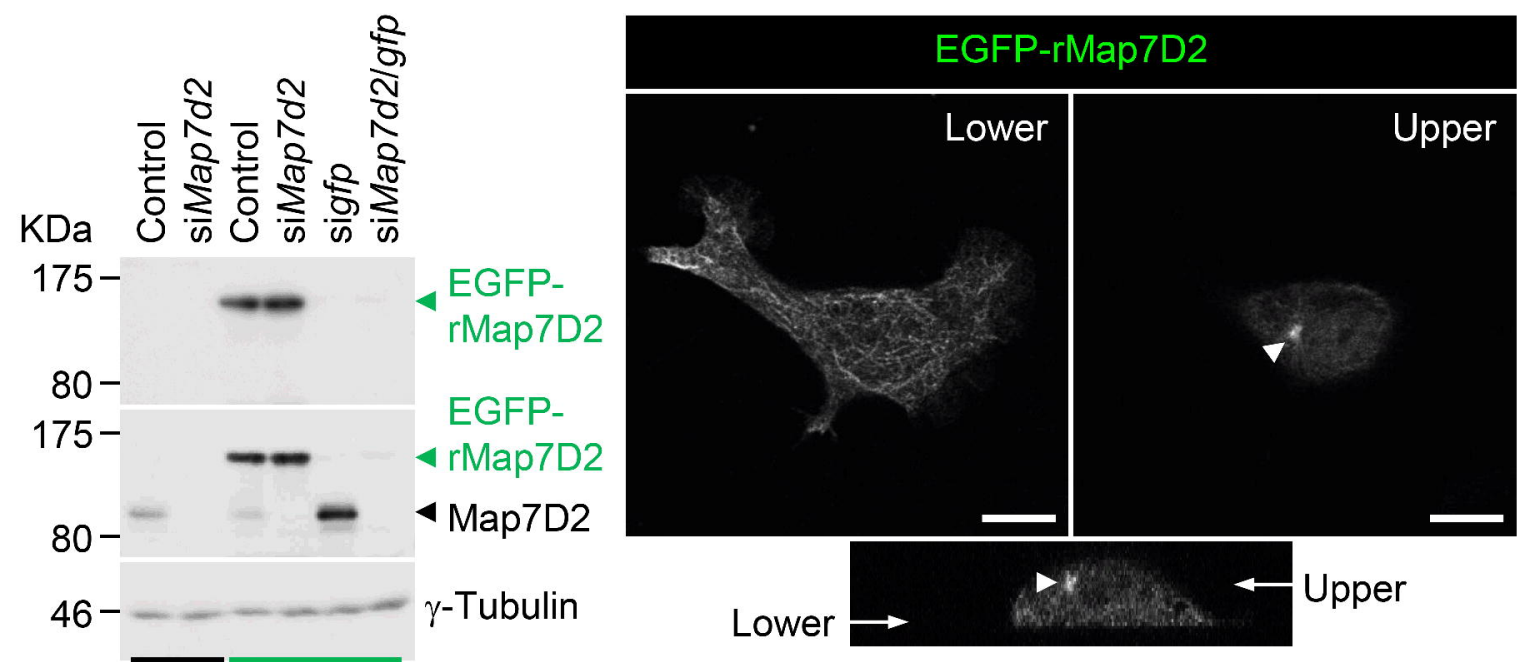

Parental +EGFP-rMap7D2 
Kikuchi_Figure 4

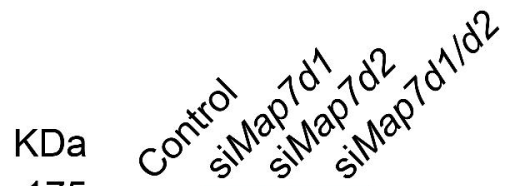

Map7D1

$80-=-\quad$ Map7D2

175- - - Clathrin HC

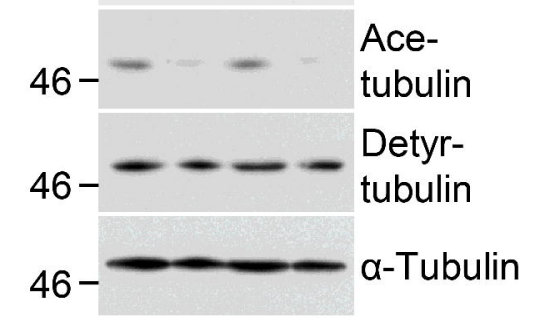

B

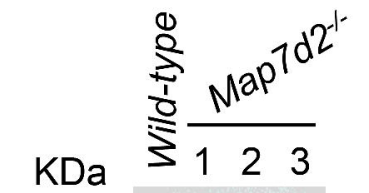

94.6- $\quad$ Map7D2

138.4 † $=\mathrm{s}=\mathrm{m}$ Map7D1 $94.6-$

45.1-_- - Actin Ace-

$45.1--1$ tubulin

$45.1----\begin{aligned} & \text { Detyr- } \\ & \text { tubulin }\end{aligned}$

45.1- $a-$ Tubulin
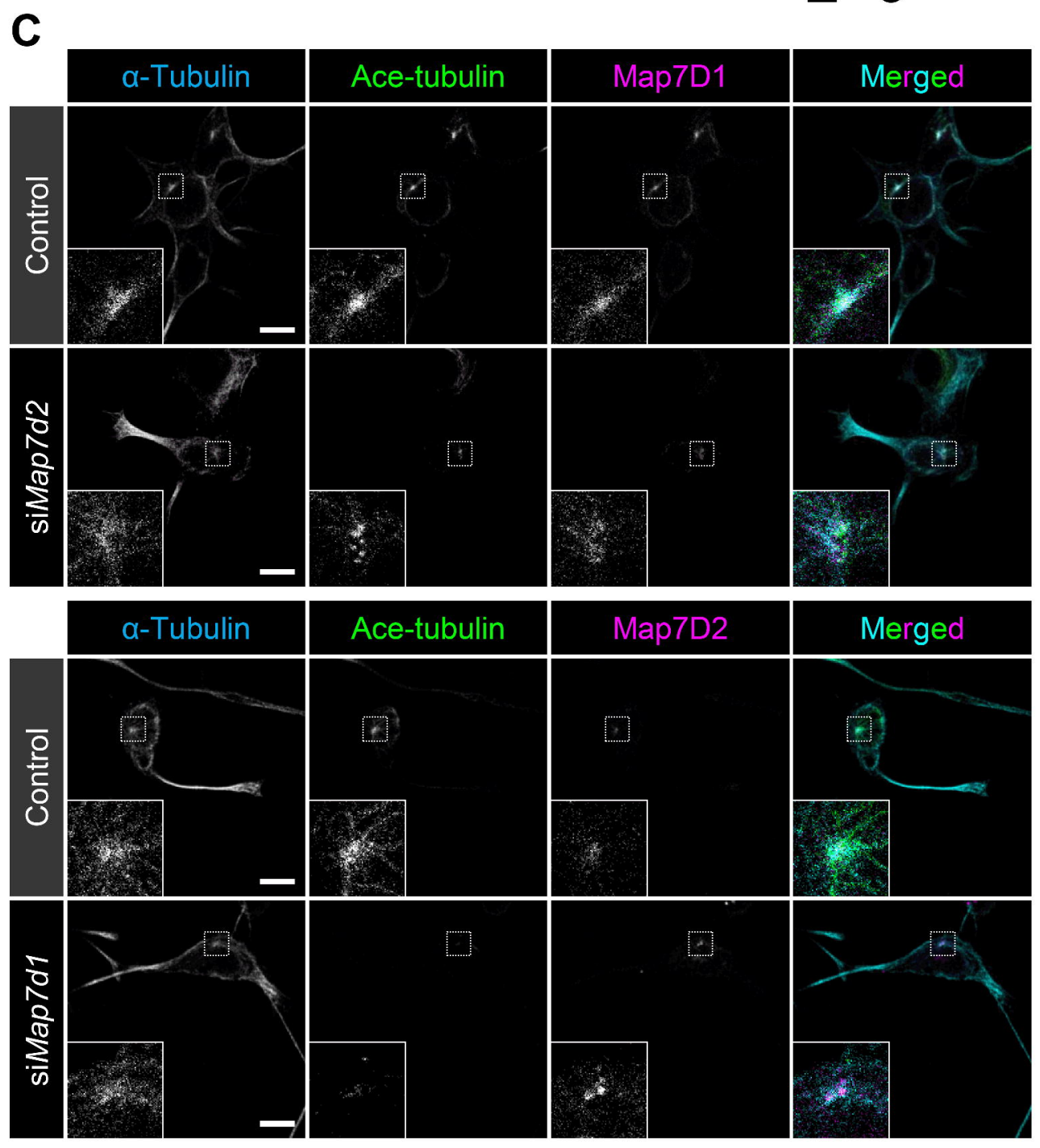

D
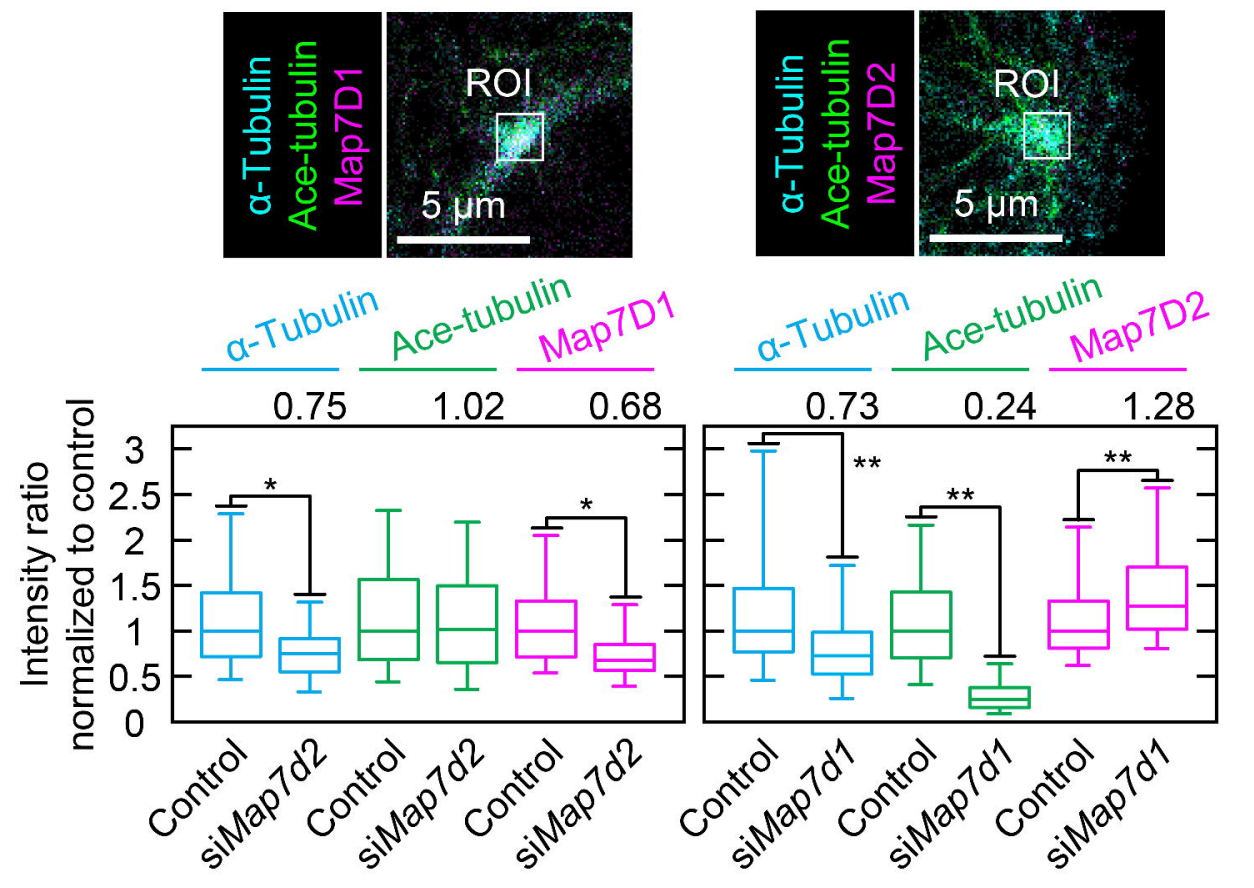
Kikuchi_Figure 5

0

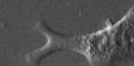

$-$
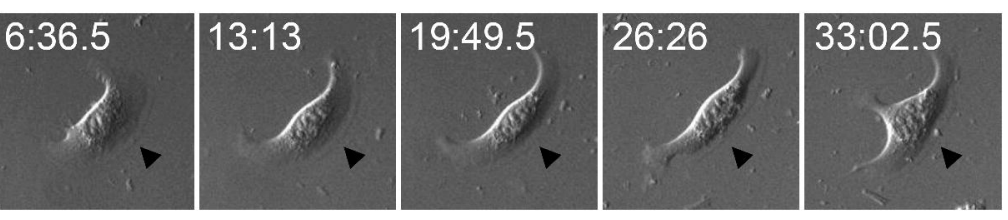

Control

siMap7d1
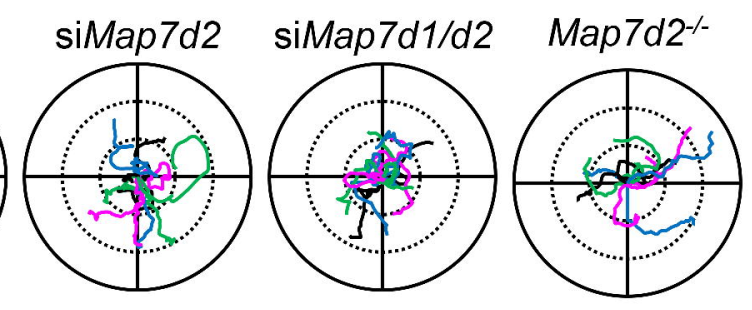

$300 \mu \mathrm{m}$
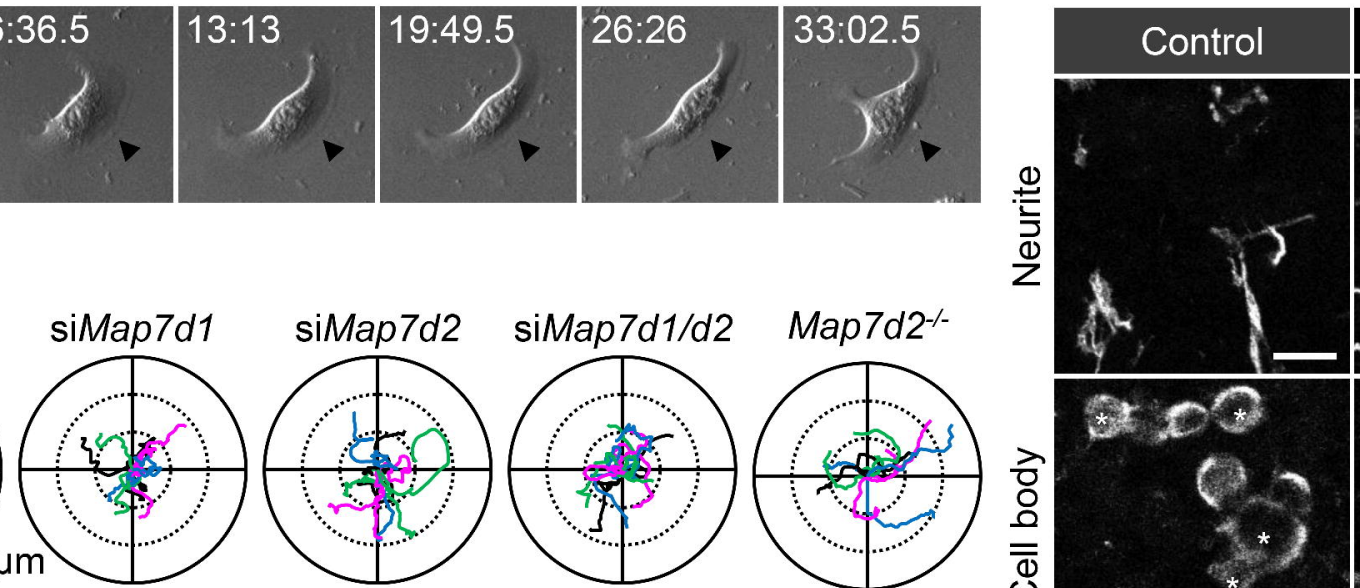

siMap $7 d 1 / d 2$
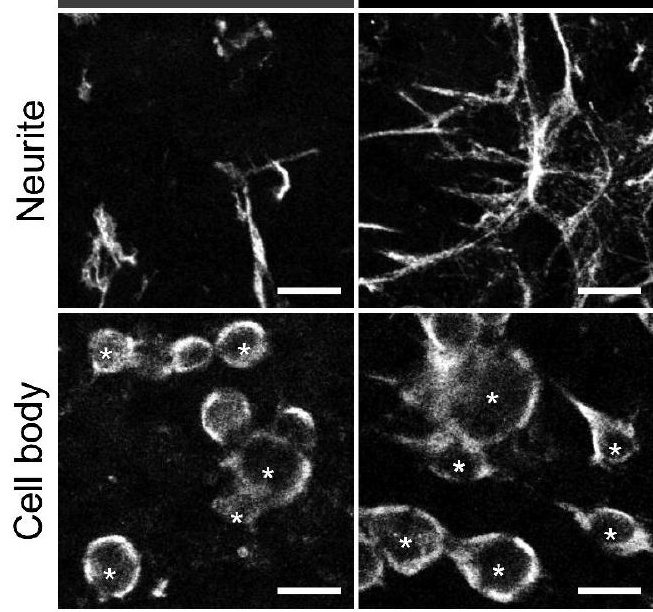

$65.3 \quad 83.5$

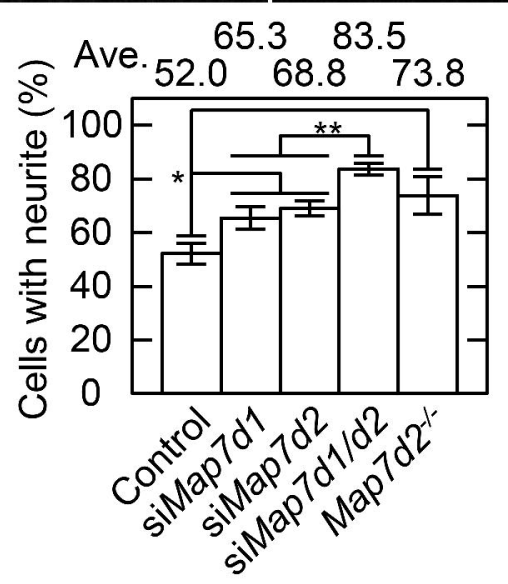

0

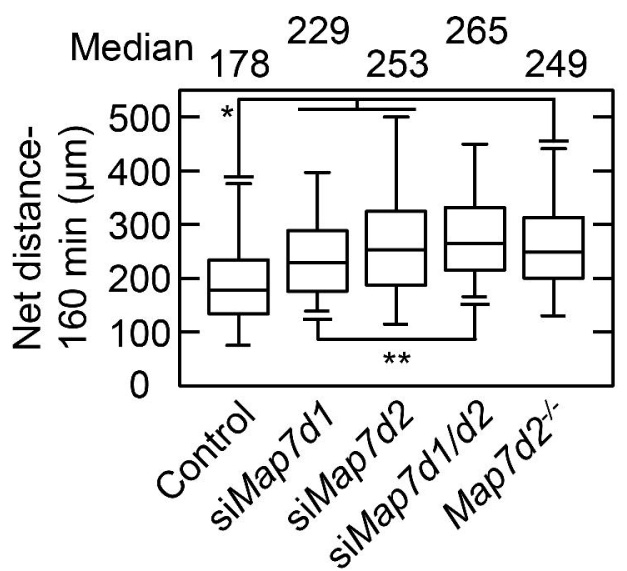

Different actions of Map7D2 and Map7D1 to stabilize MTs

MT stabilization by direct binding itself

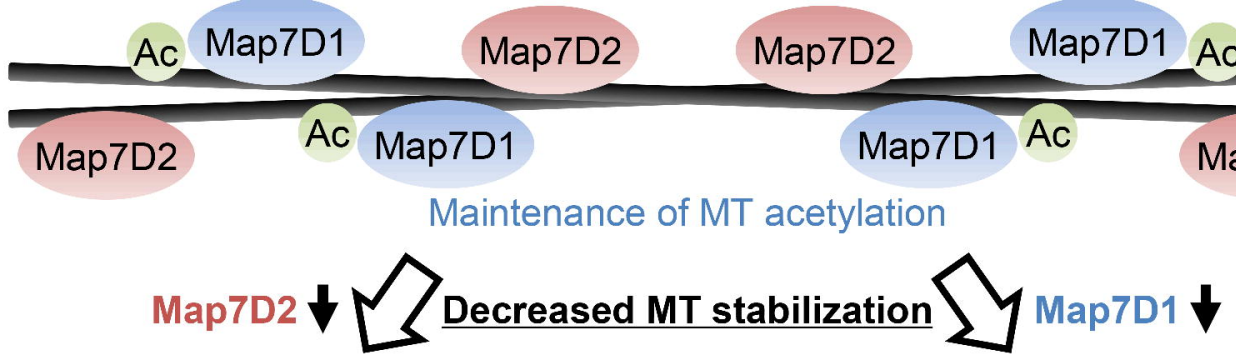

Map7D1 Ac Map7D1 Ac

MT filaments $\downarrow$

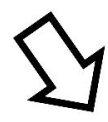

Map7D2 Map7D2 Map7D2 Map7D2

Map7D2 binding $\mathbf{4}$ MT acetylation and filaments $\downarrow$

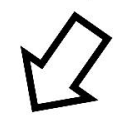

Elevated microtubule dynamics, 